\title{
Lethal and sublethal effects of polybrominated diphenyl ethers (PBDEs) for turbot (Psetta maxima) early life stage (ELS)
}

\author{
Lazhar Mhadhbi $^{1,2^{*}}$, José Fumega ${ }^{3}$, Moncef Boumaiza ${ }^{2}$ and Ricardo Beiras ${ }^{1}$ \\ 1- Toralla Marine Science Station (ECIMAT) E-36331 Vigo, Galicia, Spain. \\ 2- Hydrobiology Unit, Environmental Biomonitoring Laboratory, Faculty of Sciences, Bizerte, \\ Zarzouna 7021, Tunisia. \\ 3- Instituto Español de Oceanografía, Centro Ocenaográfico de Vigo, Cabo Estai Canido, \\ 36200 Vigo, Galicia, Spain \\ *lazhar@uvigo.es
}

\begin{abstract}
A new toxic menace, polybrominated diphenyl ethers (PBDEs), is being detected in the aquatic environment all over the world. The environmental presence of PBDEs and its entry into the environment as BDE-47 and -99 make quality aquatic toxicity data necessary to assess the aquatic hazard risk of PBDs. This study examines the effects of three PBDE-47 and -99 on embryo and larval stages of the marine flatfish turbot (Psetta maxima). The acute toxicity of the three PBDEs was examined and NOEC, LOEC, $\mathrm{LC}_{10}$ and $\mathrm{LC}_{50}$ were calculated. All tested compounds caused lethal as well as nonlethal malformations during embryo development. The effects of PBDEs in the different life stages of turbot were analysed. PBDEs seemed to be teratogenic at concentrations higher than 8.14 and $16.12 \mu \mathrm{gL}^{-1}$ for BDE-47 and -99 respectively, leading to prolonged delays in embryo development and consequent death (at $48 \mathrm{~h}$ ), as well as severe malformations and mortality of larvae. PBDEs showed a higher acute toxicity for embryo-larvae ( $\mathrm{LC}_{50}$ for lethal endpoints to embryos $27.35 \mu \mathrm{g} \mathrm{BDE}-47 \mathrm{~L}^{-1}$ and 38.28 $\mu \mathrm{g}$ BDE-99 $\mathrm{L}^{-1}$, and 14.13 and $29.64 \mu \mathrm{g} \mathrm{L}^{-1}$ for BDE-47 and -99 , respectively for larvae). Generally, the BDE-47 seemed to cause adverse effects at comparatively low dose rates, whereas much higher doses were needed to cause the same effects with BDE-99. The results of the present study show that the acute toxicity of PBDEs decreases as the degree of bromination increases, since the order of toxicity was BDE47 BDE-99. The major isomers also exhibited a clear toxic potential for turbot ELS although at high concentrations, above solubility saturation, pointing at particulate matter as the main via of uptake for those hydrophobic molecules.
\end{abstract}

Keywords: polybromodiphenyl ethers (PBDEs), ecotoxicity, lethal and sublethal effects, early life stage, turbot (Psetta maxima).

\section{Introduction}

Polybrominated diphenyl ethers (PBDEs) are widespread pollutants of increasing environmental concern due to their persistence and high bio-accumulative capacity. They are currently important components in flame retardants and other industrial processes such as electronic equipment, plastics, textiles, and building materials, thus recognized as ubiquitous environmental contaminants (de Wit 2002). Since PBDEs are polymer additives and are not chemically bound to materials, they are known to leach into the surrounding environment (de Wit 2002) and become ubiquitous 
contaminants. These compounds are not naturally biodegradable in the OECD Test (EU draft RAR 2000), they easily bio-accumulate in fatty tissues and biomagnify throughout food chains (Law et al. 2006).

These compounds are typically produced in three different formulations with varying degrees of bromination, penta-PBDE, octa-PBDE, and deca-PBDE (Alaee et al. 2003, La Guardia et al. 2006). The majority of PBDEs found in environment are 2,2',4,4'-tetrabromodiphenyl ether (BDE-47) and 2,2',4,4',5-pentabromodiphenyl ether (BDE-99). The most prevalent PBDE congeners found in maternal and cord blood are BDE-47, BDE-99, BDE-100, and BDE-154 (Guvenius et al. 2003; Mazdi et al. 2003). The constituents of penta-BDE are the most bioaccumulative and frequently encountered in common organisms, such as fishes. The order of congener dominance in these organisms is typically BDE-47 > BDE-99 > BDE-100 > BDE-154 (de Wit 2002). Recently, the constituents of Penta-BDE technical mixtures have been proposed as persistent organic pollutant (POPs) candidates since they embody all the characteristics of the Stockholm convention definition of POPs: bioaccumulation, toxicity, persistency and long-range transport potential (WWf 2005). Penta-BDE mixtures, as well as the octa-BDE mixture, have been banned in 2004 in the European Union (EU 2003).

Our objective was therefore to tests the toxicity of the three environmentally most abundant BDEs (i.e. -47, -99 and -147) on the early life stages (ELS) of turbot. Early life stages are generally considered as more sensitive than juvenile and adults. In fish early life stage tests, toxicant effects can be examined through diverse endpoints, such as hatching success, embryo-larval morphology malformations and larval survival.(Mhadhbi et al. 2010).

\section{Materials and methods}

\subsection{Biological material}

Turbot (Psetta maxima) eggs from a single stock of adults were obtained in kind from a fish hatchery (PESCANOVA, Insuiña S.L., Mougás, Galicia, Spain). Eggs were transported to the laboratory in plastic bags inside portable ice-boxes, and maintained in aquaria with running natural seawater (salinity 34\%o). Eyed eggs were acclimated to laboratory conditions for $24 \mathrm{~h}$ at $14 \pm 1^{\circ} \mathrm{C}$ (hatchery rearing temperature) before the experimental exposures to the toxicants.

\subsection{Experimental solutions and exposures}

PBDE congeners were purchased from ChemService, Inc. (Greyhound Chromatography and Allied Chemicals, UK). Stock solutions of PBDEs were dissolved in Dimethyl sulfoxide (DMSO, Sigma-Aldrich, Steinheim) and stored in amber-glass vials. The following PBDEs were selected based on their abundance in environmental samples: BDE-47, -99 and -154. For each toxicant, six concentrations in a $2 \mathrm{x}$ geometric scale, plus one control with no PBDEs added were tested, using four replicates for each condition. All treatments, including controls, contained $<0.01 \%$ (v/v) DMSO which was constant in all treatments for a given experiment. Control embryos (exposed to $1 \mu \mathrm{M}$-filtered seawater) were included as references for normal embryonic development. The final DMSO concentration was always less than $0.1 \% \mathrm{v} / \mathrm{v}$, as this was found to be non toxic in the preliminary test. At the start of the first test, the stock 
solutions were prepared in sufficient volumes and stored in the dark at room temperature. Glass vials containing at least $500 \mathrm{ml}$ of each exposure solution and 50 eggs per condition were used. Incubations were made in $1000 \mathrm{ml}$ glass beakers, to avoid losses of the tested compounds from the solutions. The experimental design followed the recommendations from OECD guidelines (OECD 1992; 1998) with the modifications indicated below. The deviations from the guidelines are the higher number of eggs per experiment and the number of reported non-lethal endpoints. All of the experiments were performed using a semi-static test with water renewal every $48 \mathrm{~h}$.

\subsection{Fish Embryo Exposure and Toxicity Assay}

Immediately after their arrival at the laboratory, within 72 hours postfertilization, the floating fertilised eggs were collected and the non-fertilised eggs at the bottom discarded. The eggs were examined under a dissecting microscope, and those embryos exhibiting normal development that had reached the blastula stage were selected for subsequent experiments. Briefly, 50 normal fertilized eggs were randomly selected and carefully distributed into exposure glass beakers containing $500 \mathrm{ml} \mathrm{FSW}$ and spiked with the test solutions. Treatments were incubated per quadruplicate in an isothermal room $\left(18 \pm 1^{\circ} \mathrm{C}\right)$, in dark. Control beakers were similarly set up. Neither food nor aeration was provided during the bioassays. Eggs were transferred into each beaker from the lowest to the highest concentration to minimize the risk of crosscontamination. The effects of the toxicants on turbot embryos and larvae were observed daily throughout the 6-day exposure period (from 0 to $2 \mathrm{~d}$ embryonic exposure and from 2 to $6 \mathrm{~d}$ larval exposure). The number of dead eggs/embryos was recorded $48 \mathrm{~h}$ after incubation. Hatching was defined as the rupture of the egg membrane, partially as well as fully hatched larvae was counted as hatched. Survival and malformation of larvae were observed and recorded every day after hatching. Mortality was identified by coagulation of the embryos, missing heartbeat, failure to develop somites and a non-detached tail. Sublethal endpoints recorded included embryo malformation and hatching success.

\subsection{Statistical analyses}

Differences between treatments were tested for significance by means of oneway analysis of variance (ANOVA). When differences among groups were significant, the Dunnett's test was employed to compare the control group to each of the experimental groups for calculation of No observed Effect Concentration (NOEC) and Lowest Observed Effect Concentration (LOEC). Statistical significance was set at $\mathrm{p} \leq$ 0.05. The $\mathrm{LC}_{10}$ and $\mathrm{LC}_{50}$ and their $95 \%$ confidence intervals $(95 \mathrm{CI})$ were calculated according to the Probit method after normalizing data to the mean control response using Abbot's formula (Emmens 1948). The results of the experiments conducted in the dark and under fluorescent light were analyzed using two-way ANOVA. Statistical analyses were conducted using the SPSS version 17.0 statistical software.

\section{Results}

The measured exposure concentrations of BDE-47 and -99 were lower to the nominal values. Exposure to PBDEs compounds led to non-lethal and lethal malformations in the early life stages of turbot according to a sigmoidal dose-response pattern shown in Fig. 1. PBDEs caused a significant decrease in embryo mortality. 


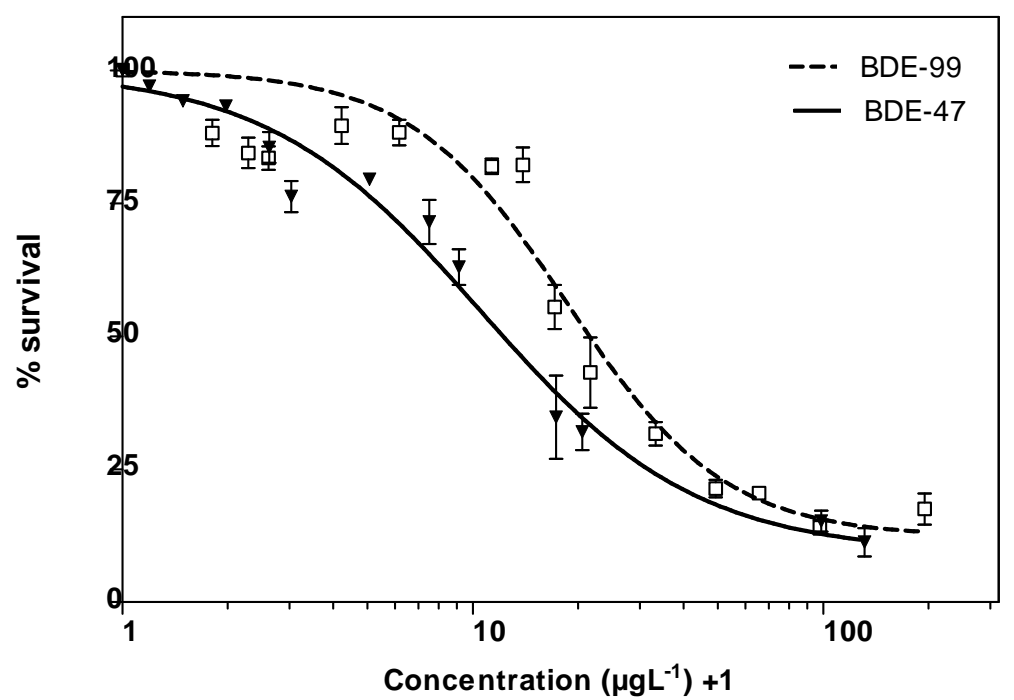

Fig. 1 Dose-effect curves of lethal malformations of turbot larval caused by BDE-47 and -99 154 at $96 \mathrm{~h}$ (mean value standard \pm error).

Surviving organisms suffered a significant decrease in success hatching, malformations (embryos), pericardial oedema and skeletal deformations (larvae), but with no all organisms dying at $96 \mathrm{hph}$. The controls showed a normal embryo-larvae development. Embryo and larvae mortality in controls was always below 10\%, as required for test validity. For concentrations up to $19.53 \mu \mathrm{g}$ BDE-47 $\mathrm{L}^{-1}$ and $32.25 \mu \mathrm{g}$ BDE-99 $\mathrm{L}^{-1}$ no significant difference in hatching success was observed for any exposure group of eggs. At higher concentrations a significant difference in hatching success was observed. In the controls and in the group with the lowest PBDEs concentrations (i.e. 0.19, 0.49, $1.63 \mu \mathrm{g} \mathrm{L}^{-1}$ BDE-47 and 0.8, 1.61, 3.22, 5.16, $12.9 \mu \mathrm{g} \mathrm{L}^{-1}$ BDE-99) embryolarvae presented a well-developed head, body and tail. For those concentrations, effects were noticed although not statistically significant. However, increasing concentrations consistently caused increasing levels of effects $(p<0.05)$. PBDE-induced morphological abnormalities in embryo-larval development are illustrated in figure 2 including no rupture of the eggs membrane, yolk sac alterations, pericardial edema and skeletal deformities. After 96 hours post-hatching (hph), the larvae exposed higher concentrations (i.e. 19.53, 97.65, $130.2 \mu \mathrm{g} \mathrm{BDE}-47 \mathrm{~L}^{-1}$ and 21.64, 32.25, 48.37, 96.75, $193.5 \mu \mathrm{g}$ BDE-99 $\mathrm{L}^{-1}$ ) presented several developmental defects, including pericardial edema and skeletal deformities. No Observed Effect Concentration (NOEC), Lowest Observed Effect Concentration (LOEC), $\mathrm{LC}_{10}$ and $\mathrm{LC}_{50}$ and their $95 \%$ confidence intervals (CI) of all PBDEs were calculated for both, embryogenesis and larval stage of turbot (Table 1). 


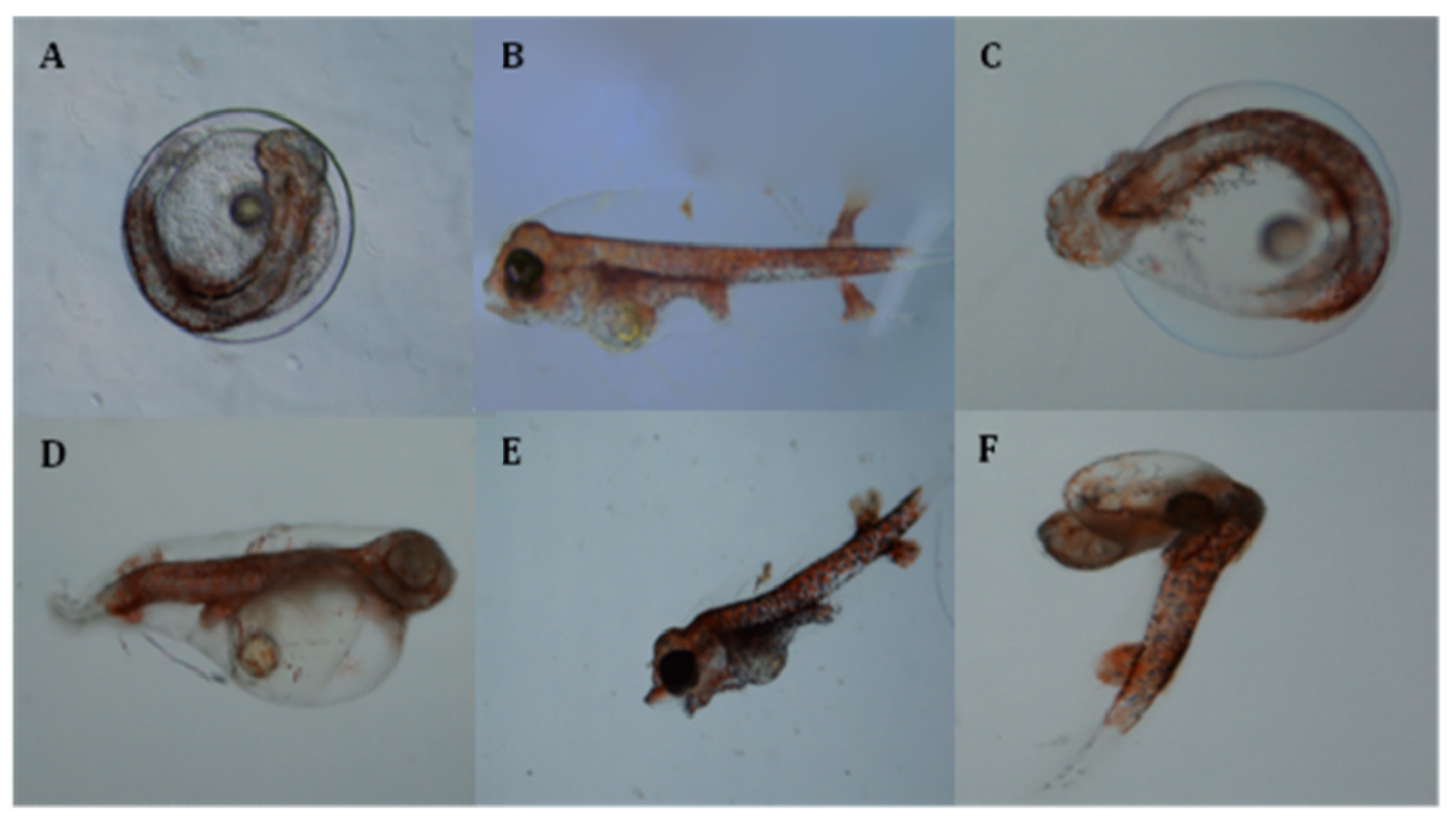

Fig 2. Morphological abnormalities of turbot embryos larvae exposed to PBDEs compounds: (A) Normal embryo, (B) Normal larvae, (C) no rupture of the eggs membrane, (D) yolk sac alterations, (E) pericardial edema, (F) skeletal deformities.

Table 1 Toxicity of PBDEs (47 and 99) for turbot embryos larvae exposed as NOEC, LOEC, $\mathrm{LC}_{10}$ and $\mathrm{LC}_{50}\left[\mu \mathrm{g} \mathrm{L}^{-1}\right]$ and their $95 \%$ confidence intervals (CI).

\begin{tabular}{lllll}
\hline & \multicolumn{2}{l}{ (48h) hatching success } & \multicolumn{2}{l}{ (96h) Larval survival } \\
\cline { 2 - 5 } & BDE-47 & BDE-99 & BDE-47 & BDE-99 \\
\hline NOEC & 2.03 & 3.22 & 0.49 & 1.61 \\
LOEC & 4.07 & 5.16 & 1.63 & 3.22 \\
LC $_{10}$ & 4.87 & 7.17 & 2.31 & 5.55 \\
$95 \%$ IC & $6.86-29.03$ & $12.94-29.7$ & $0.07-0.84$ & $0.23-9.77$ \\
LC & 27.35 & 38.28 & 14.13 & 29.64 \\
$95 \%$ IC & $18.12-60.4$ & $57.1-221.9$ & $13.8-21.19$ & $22.38-40.27$ \\
\hline
\end{tabular}

\section{Discussion}

PBDE have been increasingly used throughout the world and they have a high potential to affect various aquatic organisms through unexpected modes of action. While most research so far has examined endocrine disruption of PBDEs in fishes, there is very limited information of acute toxicity of PBDEs such as BDE-47 and BDE-99 on the early life stage of fishes and no one in turbot. Here we provided some insight into PBDEs ecotoxicity on embryo-larvae of turbot.

Ours results show that PBDEs can induce health effects in fishes via water phase, which is consistent with previous studies which showed effects via exposure to contaminated food and sediment (Kuiper et al. 2008). Though the tested PBDEs 
concentrations exceeded their water solubility in a number of cases, full concentrationresponses were obtained for these in the acute toxicity tests. The results showed that BDE-99 had lower NOEC/LOEC values than BDE-47 for both, embryos and larvae. Previous literature has shown that the acute toxicity of PBDEs is relatively low (Darnerud et al., 2001). So far, acute effects in fishes have not been reported in the literature, we used results presented for other groups as an approximation. Our results are in line with those observed in Nitocra spinipes copepod where the LOEC for larval development inhibition was $13 \mu \mathrm{g}$ BDE-47 $\mathrm{L}^{-1}$ and $30 \mu \mathrm{g}$ BDE-99 $\mathrm{L}^{-1}$ (Breitholtz and Wollenberger 2003).

Hatching success has been widely used as an endpoint in fish early-life stage tests. The results showed that embryos' mortality was dependent on the PBDEs concentrations with $\mathrm{LC}_{50}$ being $27.35 \mu \mathrm{g} \mathrm{L}^{-1}$ for BDE-47. The turbot embryos in our study were more sensitive to high BDE-47 concentrations than zebrafish (Danio rerio) embryos, as reported by Lema et al. (2007) and generally hatched later. For some toxicants egg's chorion may act as a barrier for embryo protection (Hallare et al. 2006), the high lipophilicity of PBDEs means the relatively high mortality of embryos reported here and in other studies (e.g. Lema et al. 2007) could be due to an incorporation of PBDEs into the egg. It also suggests that incorporated PBDE-47 may be bioavailable to induce toxicological effects even later in development (Lema et al. 2007).

The results also show that PBDEs above threshold concentrations 16.27 and $21.12 \mu \mathrm{g} \mathrm{L}^{-1}$ for BDE-47 and -99 respectively, reduced the hatching success and caused many deformities in turbot. One-way ANOVA using ranks showed that treatment had a statistically significant effect $(\mathrm{p}<0.05)$ on a gross abnormalities. The most pronounced effects of BDE-47 were abnormal skeletal formations while for BDE-99 was pericardial edema. This is consistent with morphological abnormalities reported for zebrafish exposures to BDE-47 during embryogenesis (Lema et al. 2007).

Significant mortality was not observed until $72 \mathrm{~h}$ after exposure to PBDEs. A significant mortality was observed at $96 \mathrm{~h}$ exposure. These results are similar to those obtained in zebrafish (Lema et al. 2007) and also marine crustaceans or algae (Key et al. 2008; Källqvist et al. 2006). Embryos exposed to high concentrations of BDE-99 in our study suffered a significantly decrease in hatching success. It also presented a high incidence of pericardial oedemas, spine malformations and increased mortality. However, the most pronounced effect in larvae exposure was abnormal skeletal formation. It has been observed that increasing all PBDEs concentrations $96 \mathrm{hph}$ produced significant effects on hatching success and mortality, deformities and malformations on larvae. There were significant differences in number of dead larvae $(P<0.05)$ between 24 and $96 \mathrm{~h}$ for all PBDEs concentrations used. This is in line with the work of Kuiper et al. (2008) whose exposure of juvenile zebrafish to PBDEs resulted in retardation of development followed by increasing mortality during the first week after hatching in animals exposed to 160 and $500 \mu \mathrm{g} \mathrm{L}^{-1}$. Our results therefore suggest that the turbot seems to be more sensitive to BDE-99 than other studied organisms.

The mortality of turbot larvae exposed to high PBDEs concentrations illustrates a high sensitivity of larvae at levels at which toxicity became apparent, consistent with 
the observations of Lema et al. (2007) and Kuiper et al. (2008). The reduced larval survival observed from our results also agree with Timme-Laragy et al. (2006) who observed reduced juvenile survival in killifish under exposure of a BDE mixture. For adult fish, the literature indicated a lack of effects (e.g. Brinbaum and Staskal, 2004; Kuiper et al. 2006). It is important to signal that the toxicity of PBDEs is similar or lower than this founded four other toxicants i.e: PCB compounds $\left(\mathrm{LC}_{50}\right.$ of PCB 126 for Solea solea ranged between 1.7 and $3.7 \mathrm{ng}$ PCB L ${ }^{-1}$ observed by Foekema et al. (2008)) and a other toxic (e.g. $\mathrm{LC}_{50}$ of $\mathrm{Cu}$ and $\mathrm{Cd}$ were 47.39 and $51.54 \mu \mathrm{g} \mathrm{L}^{-1}$, respectively (Mhadhbi et al. 2010).

In this study, serious effects of PBDEs on the early life stages of turbot were observed. Embryos were less sensitive to PBDEs than larvae may be the chorion might have protected our embryos as suggested by Frent (1991). Maybe, differences in the bioaccumulation are one reason for the differences observed in mortality. A general assumption is that lower numbered brominated congeners are more toxic than the higher ones. As a result, US-EPA has recently banned the domestic production of tetra, penta, and octa PBDEs, although importation of products containing these PBDEs is still allowed. Most of the effects observed in this study occurred at an exposure concentration of $12.9 \mu \mathrm{g} \mathrm{L}^{-1}$, which is much higher than concentrations found in the environment. Assuming that the concentrations at which visible effects caused by PBDEs in vitro are high it could at least partially reflect effects caused by PBDEs at lower concentrations in vivo. However, acute toxicity tests with turbot embryo can only be a first step for the assessment of the environmental risk of these chemicals. This is because the PBDEs form a group of highly hydrophobic and persistent chemicals that are detected in various environmental matrices such as soil, water, sediments, and biota (Källqvist et al. 2006). In addition to potential ecological concerns, it is now recognized that consumption of contaminated fish is the predominate route of exposure of PBDEs in humans (Schecter et al. 2003; 2005). The rising levels of PBDEs in aquatic organisms has raised environmental concern due in part to structural similarities with polychlorinated biphenyls (PCBs) and potential toxic effects, which may include effects on neurobehavioral development (Branchi et al. 2005) and endocrine disruption (Muirhead et al. 2006). The PBDEs thus have to be considered as very toxic to aquatic organisms (Wollenberger et al. 2003).

\section{Conclusions}

Turbot fish are an excellent model for the study of ecotoxicity of contaminants in water. The effects of PBDEs in the different life stages of turbot were analysed. PBDEs seemed to be teratogenic to embryo -larval stage at concentrations higher than $8.14 \mu \mathrm{gBDE}-47 \mathrm{~L}^{-1}$ and $12.9 \mu \mathrm{gBDE}-99 \mathrm{~L}^{-1}$, leading to malformations and no success hatching in embryo development and consequent death (at $48 \mathrm{~h}$ ). At $10 \mu \mathrm{gL}^{-1}$ PBDEs caused a significant decrease in embryo mortality. Surviving organisms suffered a significant decrease in success hatching, malformations (embryos), pericardial oedema and skeletal deformations (larvae), but with no all organisms dying at $96 \mathrm{hph}$.

In summary, our results pointed out that the toxicity of PBDEs probably declines during bromination, the order of toxicity was BDE-47 BDE-99, but does not vanish 
since the major isomer exhibit a clear toxic potential for turbot embryo-larvae although probably with a very high concentration.

\section{Acknowledgments}

The authors gratefully acknowledge the cooperation of all the workers and personnel at the ECIMAT and Laboratory of Marine ecology, especially those workers who willingly participated in the hatchery Damián Costas and Arantxa Martinez Casal. The authors would like to thank David Chavarrias Lazaro production manager of hatchery PESCANOVA S.L (Insuiña Mougás, Galicia, Spain). This study was financially supported by the MAE-PCI (Ministry of Foreign Affairs, Spain) And the Ministry of higher education, scientific research and technology in Tunisia.

\section{References}

Alaee M, Arias P, Sjödin A, Bergman Å (2003) An overview of commercially used brominated flame retardants (BFRs) and their applications, changes in their use pattern in different countries/regions over time and possible modes of release. Environ Int 29:683-689

Branchi I, Capone F, Vitalone A, Madia F, Santucci D, Alleva E, Costa LG (2005) Early developmental exposure to BDE 99 or aroclor 1254 affects neurobehavioural profile: interference from the administration route. Neurotoxicology 26:183-192

Breitholtz M, Wollenberger L (2003) Effects off PBDEs on development, reproduction and population growth rate of harpacticoid copepod Nitocra spinipes. Aquatic Toxicology 64:85-96

Darnerud PO, Eriksen GS, Johannesson T, Larsen PB, Viluksela M (2001) Polybrominated diphenyl ethers: occurrence, dietary exposure, and toxicology, Environ. Health Perspect. 109: 49-68

de Wit CY (2002) An overview of brominated flame retardants in the environment. Chemosphere 46:583-624

Emmens CW (1948) Principles of Biological Assay. Chapman and Hall Ltd., London

EPA (2004) bans manufacture of penta- and octa-BDE. Chem. Week 166 (41) 28

EU (2003) Directive 2003/11/EC of the European Parliament and of the Council of 2 February 2003 amending the 24th time Council Directive 76/769/EEC relating to restrictions on the marketing and use of certain dangerous substances and preparations (pentabromodiphenyl ether, octabromodiphenyl ether). Official Journal of the European Union. 15.2.2003

EU draft RAR (2000) EU Risk Assessment Reports for bis (pentabromophenyl) ether (decabromodiphenyl ether) CAS 1163-19-5, EC Chemical Number R013, RAR draft number as stated. Available at: http://ecb.jrc.it/existing-chemicals/.

Fent, K (1991). Bioconcentration and elimination of tributyltin chloride by embryos and larvae of minnows Phoxinus phoxinus. Aquat. Toxicol. 20, 147-158.

Foekema EM, Deerenberg CM, Murk AJ (2008) Prolonged ELS test with the marine flatfish sole (Solea solea) shows delayed toxic effects of previous exposure to PCB 126. Aquatic Toxicology 90:197203

Guvenius DM, Aronsson A, Ekman-Ordeberg G, Bergman A, Norén K (2003) Human prenatal and postnatal exposure to polybrominated diphenyl ethers, polychlorinated biphenyls, polychlorobiphenylols, and pentachlorophenol. Environ Health Perspect 111(9):1235-1241

Hallare A, Nagel K, Köhler H-R, Triebskorn R (2006) Comparative embryotoxicity and proteotoxicity of three carrier solvents to zebrafish (Danio rerio) embryos Ecotoxicol. Environ Saf 63:378-388

Källqvist T, Grung M, Tollefsen KE (2006) Chronic tox- icity of 2,4,29,49tetrabromodiphenyl ether on the marine alga Skeletonema Costatum and the crustacean Daphnia magna. Environ. Toxicol. Chem 25 (6):1657-1662

Key PB, Chung KW, Hoguet J, Shaddrix B, Fulton MH (2008) Toxicity and physiological effects of brominated flame retardant PBDE-47 on two life stages of grass shrimp, Palaemonetes pugio. Sci of Total Environ 399:28-32

Kuiper RV, Murk AJ, Leonards PEG, Grinwis GCM, van den Berg M, Vos JG (2006) In vivo and in vitro Ah-receptor activation by commercial and fractionated pentabromodiphenylether using zebrafish (Danio rerio) and the DR-CALUX assay. Aquat. Toxicol 79:366-375 
Kuiper RV, Vethaak AD, Cantón RF, Anselmo H, Dubbeldam M, van den Brandhof EJ, Leonards PEG, Wester PW, van MdB (2008) Toxicity of analytically cleaned pentabromodiphenylether after prolonged exposure in estuarine European flounder (Platichthys flesus), and partial life-cycle exposure in fresh water zebrafish (Danio rerio), Chemosphere 73:195-202

La Guardia MJ, Hale RC, Harvey E (2006) Detailed polybrominated diphenyl ether (PBDE) congener composition of the widely used penta-, octa-, and deca-PBDE technical flame-retardant mixtures. Environ. Sci. Technol 40: 6247-6254

Law RJ, Allchin CR, de Boer J, Covaci A, Herzke D, Lepom P, Morris S, Tronczynski J, de Wit CA (2006) Levels and trends of brominated flame retardants in the European environment. Chemosphere 64: 187-208

Lema SC, Schultz I, Scholz N, Incardona J, Swanson P (2007) Neural defects and cardiac arrhythmia in fish larvae following embryonic exposure to 2,2',4,4'-tetrabromodiphenyl ether (PBDE-47). Aquat Toxicol 82:296-307

Mhadhbi L, Boumaiza M, Beiras R (2010) A standard ecotoxicological bioassay using early life stages of the marine fish Psetta maxima. Aquat. Living Resour. DOI: 10.1051/alr/2010

Muirhead EK, Skillman AD, Hook SE, Schultz IR (2006) Oral exposure of PBDE-47 in fish: toxicokinetics and reproductive effects in Japanese medaka (Oryzias latipes) and fathead minnows (Pimephales promelas). Environ Sci Technol 40:523-528

OECD (1992) OECD Guidelines for the Testing of Chemicals. Section 2: Effects on Biotic Systems Test No. 210: Fish, Early-Life Stage Toxicity Test. Paris, France: Organization for Economic Cooperation and Development

OECD (1998) OECD Guidelines for the Testing of Chemicals. Section 2: Effects on Biotic Systems Test No. 212: Fish, Short-term Toxicity Test on Embryo and Sac-Fry Stages. Paris, France: Organization for Economic Cooperation and Development

Schecter A, Paepke O, Joseph JE, Tung KC (2005) Polybrominated diphenyl ethers (PBDEs) in U.S. computers and domestic carpet vacuuming: possible sources of human exposure. J Toxicol Environ Health A 68:501-513

Timme-Laragy AR, Levin ED, Di Giulio RT (2006) Developmental and behavioral effects of embryonic exposure to the polybrominated diphenylether mixture DE-71 in the killifish (Fundulus heteroclitus). Chemosphere 62:1097-1104

Wollenberger L, Dinan L, Breitholtz M (2005) Brominated flame retardants: activities in a crustacean development test and in an ecdysteroid screening assay. Environmental Toxicology and Chemistry 24 (2):400-407

WWF (2005) World Wide Found. Stockholm Convention "New POPs": Screening additional POPs candidates. April 2005, 38 p 